\title{
ON THE NUMBER OF $p$-ELEMENTS IN A FINITE GROUP
}

\author{
PIETRO GHERI \\ This work is dedicated to the memory of Carlo Casolo. \\ His knowledge, his curiosity, his humility and his humanity were an example to all of his students and friends.
}

\begin{abstract}
In this paper we study the ratio between the number of $p$-elements and the order of a Sylow $p$-subgroup of a finite group $G$. As well known, this ratio is a positive integer and we conjecture that, for every group $G$, it is at least the $\left(1-\frac{1}{p}\right)$-th power of the number of Sylow $p$-subgroups of $G$. We prove this conjecture if $G$ is $p$-solvable. Moreover, we prove that the conjecture is true in its generality if a somewhat similar condition holds for every almost simple group.
\end{abstract}

\section{INTRODUCTION}

Let $G$ be a finite group and $p$ be a prime dividing the order of $G$. Moreover, let

$$
\mathfrak{U}_{p}(G)=\bigcup_{P \in \operatorname{Syl}_{p}(G)} P
$$

be the set of $p$-elements of $G$.

A celebrated theorem of F.G. Frobenius ([3]) states that if $P$ is a Sylow $p$-subgroup of $G$, then $|P|$ divides $\left|\mathfrak{U}_{p}(G)\right|$. We will call the positive integer $\left|\mathfrak{U}_{p}(G)\right| /|P|$ the $p$-Frobenius ratio of $G$.

The number of $p$-elements of a finite group is a fundamental invariant in finite group theory. Several different proofs of Frobenius' theorem have been given (see, for example, [6] and [10]). Moreover in [11, Theorem 15.2] it is proven that the $p$-Frobenius ratio in a finite group of Lie type is equal to the size of a Sylow $p$-subgroup.

Nevertheless, it is still unknown if the Frobenius ratio has a combinatorial meaning.

It is clear that the $p$-Frobenius ratio is 1 if and only if $G$ contains a normal Sylow $p$ subgroup. In [8], it is proven with a nice and easy argument that if the $p$-Frobenius ratio is not 1 , then it must be greater or equal than $p$.

In this paper, we focus on the search for "good" bounds for the $p$-Frobenius ratio in terms of the number $n_{p}(G)$ of Sylow $p$-subgroups of $G$.

Of course, a trivial upper bound is obtained when every pair of Sylow p-subgroups of $\mathrm{G}$ has trivial intersection, so that, given a Sylow $p$-subgroup $P$ of $G$,

$$
\frac{\left|\mathfrak{U}_{p}(G)\right|}{|P|} \leq n_{p}(G)-\frac{n_{p}(G)-1}{|P|} \leq n_{p}(G) .
$$

It is not hard to find examples of sequences of groups that show that a lower bound on the $p$-Frobenius ratio cannot be linear in $n_{p}(G)$. We state the following conjecture.

Conjecture A. Let $G$ be a finite group, $p$ be a prime dividing $|G|$ and $P$ a Sylow $p$ subgroup of $G$. Then

$$
\frac{\left|\mathfrak{U}_{p}(G)\right|}{|P|} \geq n_{p}(G)^{1-\frac{1}{p}}
$$


We will show in Example 2.5 that this bound is "asymptotically tight". We show that Conjecture 1 is true for $p$-solvable groups. Namely, we prove the following.

Theorem A. Let $G$ be a finite $p$-solvable group and $P$ be a Sylow p-subgroup of $G$. If $n_{p}(G)$ denotes the number of Sylow p-subgroups in $G$, then

$$
\frac{\left|\mathfrak{U}_{p}(G)\right|}{|P|} \geq n_{p}(G)^{\frac{p-1}{p}} .
$$

Inspired by the proof of Theorem $\mathrm{A}$, we show that a sufficient condition for Conjecture 1 to be true in general is that

$$
\left(\prod_{x \in P} \lambda_{G}(x)\right)^{1 /|P|} \leq n_{p}(G)^{\frac{1}{p}}
$$

where for every $p$-element $x$ of $P, \lambda_{G}(x)$ denotes the number of Sylow $p$-subgroups of $G$ containing $x$.

For this condition we give a reduction to almost simple groups.

Theorem B. Inequality (3) holds for every finite group if and only if it holds for every finite almost simple group.

One of the most important tools used here is the so-called Wielandt's subnormalizer (see Definition 2.1), which is related to the number of $p$-elements (see Lemma 2.3). This connection is mainly due to the works of C. Casolo on subnormalizers ([1], [2]).

Another fundamental tool for the proof of our result is a theorem by G. Navarro and N. Rizo, concerning the number of fixed points in a coprime action of a $p$-group.

Throughout the paper $G$ will be a finite group and $p$ a prime dividing $|G|$. Also, for all $x \in G$ we denote with $x^{G}$ the conjugacy class of $x$ in $G$.

\section{The P-SOlVABle CASE}

In this section we prove Theorem $\mathrm{A}$. First of all we introduce the we introduce the concept of subnormalizer, whose definition (see [7, pag. 238]) is inspired by the celebrated Wielandt's subnormality criterion, which says that a subgroup $H$ of $G$ is subnormal in $G$ if and only if $H$ is subnormal in $\langle H, g\rangle$ for every $g \in G$.

Definition 2.1. Let $H$ be a subgroup of $G$. The subnormalizer of $H$ in $G$ is the set

$$
S_{G}(H)=\{g \in G \mid H \unlhd \unlhd\langle H, g\rangle\} .
$$

where $\unlhd \unlhd$ means “is subnormal in".

A useful link between subnormalizers and the number of p-elements in a finite group (see Lemma 2.3) is established by using a beautiful theorem by C. Casolo In order to state this theorem we introduce some notation. Let $H$ be a $p$-subgroup of $G$ and $P$ be a Sylow $p$-subgroup of $G$. We write $\lambda_{G}(H)$ for the number of Sylow $p$-subgroups of $G$ containing $H$ and $\alpha_{G}(H)$ for the number of $G$-conjugates of $H$ contained in $P$ (note that this number does not depend on the Sylow subgroup $P$ we are considering). When $H=\langle x\rangle$ is a cyclic subgroup, we simply write $S_{G}(x)$ and $\lambda_{G}(x)$, in place of $S_{G}(\langle x\rangle)$ and $\lambda_{G}(\langle x\rangle)$. In a similar fashion, we write $\alpha_{G}(x)$ for the number of $G$-conjugates of the element $x$ contained in $P$. We thus have that

$$
\alpha_{G}(x)=\alpha_{G}(\langle x\rangle)\left|N_{G}(\langle x\rangle)\right| /\left|C_{G}(x)\right| .
$$

We can now state the aforementioned theorem by C. Casolo. 
Theorem 2.2 ([1],[2]). Let $H$ be a p-subgroup of $G$. Then the following holds and $P$ be a Sylow p-subgroup of $G$.

a)

$$
\left|S_{G}(H)\right|=\lambda_{G}(H)\left|N_{G}(P)\right|=\alpha_{G}(H)\left|N_{G}(H)\right| .
$$

b) If $G$ is $p$-solvable and $\mathcal{M}$ is the set of all $p^{\prime}$-factors in a given normal $\left\{p, p^{\prime}\right\}$-series of $G$, then

$$
\left|S_{G}(H)\right|=|P| \prod_{U / V \in \mathcal{M}}\left|C_{U / V}(H V / V)\right| .
$$

A first easy application of this result is a formula that expresses the number of $p$ elements in $G$ in terms of the orders of the subnormalizers of the cyclic subgroups of a Sylow $p$-subgroup of $G$.

Lemma 2.3. Let $P$ be a Sylow p-subgroup of $G$. We have

$$
\left|\mathfrak{U}_{p}(G)\right|=\sum_{x \in P} \frac{|G|}{\left|S_{G}(x)\right|} .
$$

Proof. In the sum

$$
\sum_{x \in P}\left|x^{G}\right|
$$

every class of $p$-elements is involved and its contribution is repeated as many times as the cardinality $\left|x^{G} \cap P\right|=\alpha_{G}(x)$. Hence

$$
\left|\mathfrak{U}_{p}(G)\right|=\sum_{x \in P} \frac{\left|x^{G}\right|}{\alpha_{G}(x)}=\sum_{x \in P} \frac{|G|}{\alpha_{G}(x)\left|C_{G}(x)\right|}=\sum_{x \in P} \frac{|G|}{\left|S_{G}(x)\right|},
$$

by part $a$ ) of Theorem 2.2 and formula (4).

We now turn to the proof of Theorem A Another fundamental tool that we are going to use in the proof is the following formula proved by Navarro and Rizo.

Theorem 2.4 ([9]). Suppose that $P$ is a $p$-group acting on a $p^{\prime}$-group $G$. Then

$$
\left|C_{G}(P)\right|=\left(\prod_{x \in P} \frac{\left|C_{G}(x)\right|}{\left|C_{G}\left(x^{p}\right)\right|^{1 / p}}\right)^{\frac{p}{(p-1)|P|}} .
$$

We can now prove Theorem $\mathrm{A}$,

Proof of Theorem $A$ By Lemma 2.3 we have that the $p$-Frobenius ratio of $G$ is the arithmetic mean of the ratios

$$
\frac{|G|}{\left|S_{G}(x)\right|}
$$

when $x$ runs across $P$. By the Arithmetic-Geometric Mean Inequality, we get

$$
\frac{\left|\mathfrak{U}_{p}(G)\right|}{|P|}=\frac{1}{|P|}\left(\sum_{x \in P} \frac{|G|}{\left|S_{G}(x)\right|}\right) \geq\left(\prod_{x \in P} \frac{|G|}{\left|S_{G}(x)\right|}\right)^{1 /|P|} .
$$

Since $G$ is $p$-solvable we can take a normal $\left\{p, p^{\prime}\right\}$-series, whose set of $p^{\prime}$-factors we call $\mathcal{M}$. Then, by part $b$ ) of Theorem 2.2 we have for all $x \in P$

$$
\frac{|G|}{\left|S_{G}(x)\right|}=\frac{|G| /|P|}{\prod_{U / V \in \mathcal{M}}\left|C_{U / V}(V x)\right|}=\prod_{U / V \in \mathcal{M}} \frac{|U / V|}{\left|C_{U / V}(V x)\right|} .
$$


We insert this last term in (5) and swap the products to get

$$
\begin{aligned}
\frac{\left|\mathfrak{U}_{p}(G)\right|}{|P|} & \geq\left(\prod_{U / V \in \mathcal{M}}\left(\prod_{x \in P} \frac{|U / V|}{\left|C_{U / V}(x V)\right|}\right)\right)^{1 /|P|} \\
& =\left(\prod_{U / V \in \mathcal{M}}\left|\frac{U}{V}\right|^{|P|}\left(\prod_{x \in P} \frac{1}{\left|C_{U / V}(x V)\right|}\right)\right)^{1 /|P|} .
\end{aligned}
$$

Now for all $U / V \in \mathcal{M}, P$ is a $p$-group that acts on the $p^{\prime}$-group $U / V$. We can then apply Theorem 2.4 and use the trivial inequality $\left|C_{U / V}\left((x V)^{p}\right)\right| \leq|U / V|$, so that we have

$$
\begin{aligned}
\prod_{x \in P} \frac{1}{\left|C_{U / V}(x V)\right|} & =\left(\prod_{x \in P} \frac{1}{\left|C_{U / V}\left((x V)^{p}\right)\right|^{1 / p}}\right) \frac{1}{\left|C_{U / V}(P)\right|^{|P|(p-1) / p}} \\
& \geq\left(\frac{1}{|U / V|^{|P| / p}}\right) \frac{1}{\left|C_{U / V}(P)\right|^{|P|(p-1) / p}}
\end{aligned}
$$

and so

$$
\frac{\left|\mathfrak{U}_{p}(G)\right|}{|P|} \geq\left(\prod_{U / V \in \mathcal{M}} \frac{|U / V|}{\left|C_{U / V}(P)\right|}\right)^{\frac{p-1}{p}}=\left(\frac{|G|}{\left|S_{G}(P)\right|}\right)^{1-\frac{1}{p}},
$$

again by part $b$ ) of Theorem 2.2

Finally, we observe that for a Sylow $p$-subgroup $S_{G}(P)=N_{G}(P)$, so that

$$
\frac{\left|\mathfrak{U}_{p}(G)\right|}{|P|} \geq\left(\frac{|G|}{\left|S_{G}(P)\right|}\right)^{1-\frac{1}{p}}=\left(\frac{|G|}{\left|N_{G}(P)\right|}\right)^{1-\frac{1}{p}}=n_{p}(G)^{1-\frac{1}{p}} .
$$

It is worth mentioning that the bound in Theorem $\mathrm{A}$ is asymptotically tight in the sense specified by the following example.

Example 2.5. Let $p$ be a prime and, for $n$ a positive integer, let $P$ be an elementary abelian group of order $p^{n}$. Moreover set $\mathcal{M}$ to be the set of the maximal subgroups of $P$. Choose a prime $q$ such that $p$ divides $q-1$. Then for any $M \in \mathcal{M}$ we have that $P / M \simeq C_{p}$ acts fixed point freely as a group of automorphisms on a cyclic group $\left\langle a_{M}\right\rangle \simeq C_{q}$. We denote the image of the generator $a_{M}$ under this action by $a_{M}^{x M}$, for every $x M \in P / M$.

Since $\bigcap_{M \in \mathcal{M}} M=1$, it follows that $P$ acts faithfully on the direct product $N$ of the groups $\left\langle a_{M}\right\rangle$. To be more explicit the following map

$$
\begin{aligned}
P & \rightarrow \operatorname{Aut}(N) \\
x & \mapsto \phi_{x},
\end{aligned}
$$

where $\phi_{x}\left(a_{M}\right)=a_{M}^{x M}$, for all $M \in \mathcal{M}$ is an injective homomorphism.

We consider the semidirect product $G_{n}=N \rtimes P$. The normalizer of $P$ in $G_{n}$ is $C_{N}(P) P=P$, hence the number of Sylow $p$-subgroups of $G_{n}$ is

$$
n_{p}\left(G_{n}\right)=|N|=q^{|\mathcal{M}|}=q^{\frac{p^{n}-1}{p-1}} .
$$

In order to count the number of $p$-elements in $G_{n}$ we use the equality

$$
\left|\mathfrak{U}_{p}\left(G_{n}\right)\right|=\sum_{x \in P} \frac{n_{p}\left(G_{n}\right)}{\lambda_{G_{n}}(x)}
$$


which follows from Lemma 2.3 and part $a$ ) of Theorem 2.2. We thus have to compute $\lambda_{G_{n}}(x)$, for $x \in P \backslash\{1\}$. Using again part $a$ ) of Theorem 2.2, we have

$$
\lambda_{G_{n}}(x)=\frac{\alpha_{G_{n}}(x) n_{p}\left(G_{n}\right)}{\left|x^{G_{n}}\right|} .
$$

Now since $P$ is abelian and $G_{n}$ has a normal $p$-complement, we have $\alpha_{G_{n}}(x)=1$ and $\left|x^{G_{n}}\right|=|N| /\left|C_{N}(x)\right|$, so that $\lambda_{G_{n}}(x)=\left|C_{N}(x)\right|$. Given $x \in P \backslash\{1\}$, the centralizer of $x$ in $N$ is generated by those $a_{M}$ such that $a_{M}^{x M}=a_{M}$. Since $P / M$ acts fixed point freely on $\left\langle a_{M}\right\rangle$, this holds if and only if $x \in M$, hence

$$
C_{N}(x)=\left\langle a_{M} \mid x \in M\right\rangle .
$$

The number of maximal subgroups in $P$ containing a fixed nontrivial element is $\frac{p^{n-1}-1}{p-1}$, and so

$$
\left|C_{N}(x)\right|=q^{\frac{p^{n-1}-1}{p-1}} .
$$

We can then calculate the $p$-Frobenius ratio of $G_{n}$

$$
\begin{aligned}
\frac{\left|\mathfrak{U}_{p}\left(G_{n}\right)\right|}{|P|} & =\frac{1}{|P|} \sum_{x \in P} \frac{n_{p}\left(G_{n}\right)}{\lambda_{G_{n}}(x)} \\
& =\frac{1}{p^{n}}\left(1+\left(p^{n}-1\right) \frac{q^{\frac{p^{n}-1}{p-1}}}{q^{\frac{p^{n-1}-1}{p-1}}}\right) \\
& =\frac{1}{p^{n}}+\frac{p^{n}-1}{p^{n}} q^{p^{n-1}} .
\end{aligned}
$$

By (6) we have

$$
n_{p}\left(G_{n}\right)^{\frac{p-1}{p}}=q^{\frac{p^{n}-1}{p}} .
$$

We can now compare the two members of the inequality stated by Theorem By considering the limit

$$
\lim _{n \rightarrow \infty} \frac{\left|\mathfrak{U}_{p}\left(G_{n}\right)\right| /|P|}{n_{p}\left(G_{n}\right)^{\frac{p-1}{p}}}=\lim _{n \rightarrow \infty}\left(\frac{1}{p^{n} q^{\frac{p^{n}-1}{p}}}+\frac{p^{n}-1}{p^{n}} \frac{q^{p^{n-1}}}{q^{\frac{p^{n}-1}{p}}}\right)=q^{1 / p},
$$

we see that the $p$-Frobenius ratio of $G_{n}$ and the $\left(1-\frac{1}{p}\right)$-th power of the number of Sylow $p$-subgroups have the same asymptotic behaviour.

\section{THE GENERAL CASE}

In this section we explain why inequality (3) is sufficient for establishing Conjecture 1 and we prove Theorem $B$

Let $P$ a Sylow $p$-subgroup of $G$. Since Lemma 2.3 is true for any group, by applying the Arithmetic-Geometric Mean Inequality as in 5 , we get

$$
\frac{\left|\mathfrak{U}_{p}(G)\right|}{|P|} \geq\left(\prod_{x \in P} \frac{|G|}{\left|S_{G}(x)\right|}\right)^{1 /|P|}
$$

and, recalling Theorem 2.2, we can write

$$
\frac{\left|\mathfrak{U}_{p}(G)\right|}{|P|} \geq\left(\prod_{x \in P} \frac{n_{p}(G)}{\lambda_{G}(x)}\right)^{1 /|P|} .
$$


A sufficient condition for (2) is then

$$
\left(\prod_{x \in P} \frac{n_{p}(G)}{\lambda_{G}(x)}\right)^{1 /|P|} \geq n_{p}(G)^{\frac{p-1}{p}},
$$

that is

which is inequality (3).

$$
\left(\prod_{x \in P} \lambda_{G}(x)\right)^{1 /|P|} \leq n_{p}(G)^{\frac{1}{p}},
$$

Remark 3.1. In [4] it is proven that if $x$ is a $p$-element of $G$ which is not contained in the $O_{p}(G)$, then $\lambda_{G}(x)$ is at most $n_{p}(G) /(p+1)$. Focusing on a single element, this is the best one can get. Inequality (3), if true, would give a better bound on average, as it states that the geometric mean of the number of Sylow $p$-subgroups containing an element of a Sylow $p$-subgroup is at most the $p$-th root of the total number of Sylow $p$-subgroups.

Remark 3.2. The bound (3), if true, is best possible in a strict sense. If we compute the terms of inequality (3) for the groups $G_{n}$ defined in Example 2.5, an equality occurs.

Remark 3.3. For the proof of Theorem B, we can assume $O_{p}(G)=1$. This is because if $N$ is a normal $p$-subgroup of $G$, then, for all $x \in \mathfrak{U}_{p}(G)$, we have that $\lambda_{G}(x)=\lambda_{G / N}(x N)$, and so (3) holds for $G$ if and only if it holds for $G / N$.

First of all, we can reduce (3) to nonsolvable groups all of whose proper quotients are solvable (see Proposition 3.7). We need some technical lemmas, the first of whom is proved in [5, Lemma 3.3].

Lemma 3.4. Let $H$ be a subgroup of $G$ and $x \in H$ be a p-element. Then

$$
\frac{\lambda_{G}(x)}{n_{p}(G)} \leq \frac{\lambda_{H}(x)}{n_{p}(H)}
$$

Moreover, if $H \unlhd G$, then the equality holds.

Lemma 3.5. Let $N$ be a normal subgroup of $G, P$ be a Sylow p-subgroup of $G$ and $x$ an element of $P$. Assume that $G=N P$. Then

$$
\left|S_{G}(x)\right|=\left|S_{N}(x)\right||N P / N|
$$

Proof. If $x \in N$, the thesis follows from the fact that $\left|S_{G}(x)\right| /|G|=\left|S_{N}(x)\right| /|N|$, which can be easily derived from part $a$ ) of Theorem 2.2

We work by induction on $m$, where $|N P / N|=p^{m}$. If $m=0$, then $G=N$ and there is nothing to prove. Suppose that $m>0$ and $G \neq N\langle x\rangle$. Let $M$ be a maximal subgroup of $G$ containing $N\langle x\rangle$. Then, as $M \unlhd G$, by inductive hypothesis

$$
\left|S_{G}(x)\right|=\left|S_{M}(x)\right| p=\left|S_{N}(x)\right| p^{m-1} p .
$$

Finally, if $G=N\langle x\rangle$, we observe that, given $a \in N$ and $t$ a positive integer, $a x^{t} \in S_{G}(x)$ if and only if $\langle x\rangle$ is subnormal in $\left\langle x, a x^{t}\right\rangle=\langle x, a\rangle$, that is if and only if $a \in S_{G}(x)$. It follows that

$$
\left|S_{G}(x)\right|=\left|S_{N}(x)\right||N P / N| \text {. }
$$

Lemma 3.6. Let $N$ be a normal subgroup of $G, P$ be a Sylow p-subgroup of $G$ and $x \in P$. Then

$$
\lambda_{G}(x)=\lambda_{\frac{G}{N}}(N x) \lambda_{N P}(x) .
$$


Proof. First of all we show that given a $p$-element $x$, the value $\lambda_{N P}(x)$ is independent of the particular Sylow $p$-subgroup $P$ containing $x$. By Theorem 2.2 and Lemma 3.5, we have

$$
\lambda_{N P}(x)=\frac{\left|S_{N P}(x)\right|}{\left|N_{N P}(P)\right|}=\frac{\left|S_{N}(x)\right|}{\left|N_{N P}(P)\right|}\left|\frac{N P}{N}\right|=\frac{\left|S_{N}(x)\right|}{|N|}\left|n_{p}(N P)\right| .
$$

If $Q$ is another Sylow $p$-subgroup such that $x \in Q$, then of course $n_{p}(N P)=n_{p}(N Q)$, since $N P$ and $N Q$ are conjugated in $G$. Moreover $\left|S_{N}(x)\right|$ depends only on $N$ and $x$.

Now let $\Delta_{G}^{x}$ be the set of the Sylow $p$-subgroups of $G$ containing $x$. We define the map

$$
\begin{aligned}
\Delta_{G}^{x} & \rightarrow \Delta_{\frac{G}{N}}^{x N} \\
Q & \mapsto N Q / N .
\end{aligned}
$$

For all $\tilde{Q} \in \Delta_{G}^{x}$, the fiber of $N \tilde{Q} / N \in \Delta_{\frac{G}{N}}^{x N}$ is the set of Sylow $p$-subgroups $Q$ of $G$ containing $x$ and such that $N Q=N \tilde{Q}$, that is, $\Delta_{N \tilde{Q}}^{x}$. Since we proved that $\lambda_{N \tilde{Q}}(x)$ is independent of $\tilde{Q}$, we have

$$
\lambda_{G}(x)=\left|\Delta_{G}^{x}\right|=\left|\Delta_{\frac{G}{N}}^{x N}\right|\left|\Delta_{N P}^{x}\right|=\lambda_{\frac{G}{N}}(x N) \lambda_{N P}(x) .
$$

Proposition 3.7. A counterexample of minimal order to inequality (3) is a nonsolvable group having a unique minimal normal subgroup $M$, which is nonsolvable, and such that $G=M P$, where $P$ is a Sylow p-subgroup of $G$.

Proof. Let $G$ be a counterexample of minimal order to inequality 3 and let $P$ be a Sylow $p$ subgroup of $G$. By Remark 3.3 we have that $O_{p}(G)=1$. By Theorem $G$ is nonsolvable. We show that every proper quotient of $G$ is solvable. Let $M$ be a minimal normal subgroup of $G$. By Lemma3.6, we have

$$
\begin{aligned}
\prod_{x \in P} \lambda_{G}(x) & =\prod_{x \in P} \lambda_{\frac{G}{M}}(M x) \lambda_{M P}(x) \\
& =\left(\prod_{x M \in \frac{P M}{M}} \lambda_{\frac{G}{M}}(M x)\right)^{|P \cap M|}\left(\prod_{x \in P} \lambda_{M P}(x)\right) .
\end{aligned}
$$

By the minimality of $G$, we have

$$
\prod_{x M \in \frac{P M}{M}} \lambda_{\frac{G}{M}}(M x) \leq n_{p}(G / M)^{\frac{|P M / M|}{p}} .
$$

If $M P<G$, we can again assume that the inequality is true for $M P$ and so

$$
\prod_{x \in P} \lambda_{G}(x) \leq n_{p}\left(\frac{G}{M}\right)^{\frac{|P M / M|}{p}|P \cap M|} n_{p}(M P)^{\frac{|P|}{p}}=n_{p}(G)^{\frac{|P|}{p}} .
$$

Since this is true for every minimal normal subgroup of $G$, the usual subdirect product argument gives that $G$ has a unique minimal normal subgroup $M$, which is nonsolvable and such that $G=M P$.

Referring to the notation of Lemma 3.7, $M$ is the direct product of simple groups permuted by $P$. The next easy lemma loosely bounds the number of $\langle x\rangle$-invariant Sylow $p$-subgroups of $M$, where $x \in P$, in terms of the action of $\langle x\rangle$ on the direct factors of $M$. 
Lemma 3.8. Let $M \unlhd G$ be a direct product of $k$ copies of a group $L, M=L_{1} \times \cdots \times L_{k}$, let $x \in G$ be an element that permutes the factors $L_{i}$ of $M$ and let $p$ be a prime number. Then the number of $\langle x\rangle$-invariant Sylow p-subgroups of $M$ is at most $n_{p}(L)^{s}$, where $s$ is the number of orbits of $\langle x\rangle$ on $\Delta=\left\{L_{1}, \ldots, L_{k}\right\}$.

Proof. A Sylow $p$-subgroup $Q$ of $M$ is the direct product of $k$ Sylow $p$-subgroups of $L$, $Q=Q_{1} \times \cdots \times Q_{k}$. Suppose that $Q$ is normalized by $x$. If $L_{i}=L_{j}^{x^{r}}$, for $r \in \mathbb{Z}$, then $Q_{i}=Q_{j}^{x^{r}}$ and so one has at most $n_{p}(L)$ choices for each $\langle x\rangle$-orbit in $\Delta$.

We can now prove Theorem $B$

Proof of Theorem $B$ Suppose that inequality (3) is true for all finite almost simple groups and let $G$ be a counterexemple of minimal order. By Proposition 3.7 $G=M P$ where $P$ is a Sylow $p$-subgroup of $G$ and $M$ is the unique minimal normal subgroup

$$
M=L_{1} \times \cdots \times L_{k}, L_{i} \simeq L, \forall i \in\{1, \ldots, k\} .
$$

for some nonabelian simple group $L$. Moreover $P$ acts transitively on the set $\Delta=$ $\left\{L_{1}, \ldots, L_{k}\right\}$. Since we are assuming the result for almost simple groups, we have $k>1$.

Let $Q=P \cap M$. For any subgroup $Q \leq X \leq P$ we set $m_{X}$ to be the ratio

$$
m_{X}=\frac{\left|N_{M}(X)\right|}{\left|N_{M}(P)\right|}=\frac{n_{p}(G)}{n_{p}(M X)} .
$$

The last equality holds since $n_{p}(G)=\left[M P: N_{M P}(P)\right]=\left[M: N_{M}(P)\right]$ and $n_{p}(M X)=$ $\left[M X: N_{M X}(X)\right]=\left[M: N_{M}(X)\right]$. Moreover observe that if $g \in N_{M}(P)$ and $x \in X$ then

$$
x^{g}=x[x, g] \in X(M \cap P)=X Q=X,
$$

and so $N_{M}(P) \leq N_{M}(X)$.

With a slight abuse of notation, we denote with $\lambda_{M}(x)$ the number of Sylow $p$-subgroups in $M$ normalized by $x$ even for $x \notin M$. It is easy to check that $\lambda_{M}(x)=\lambda_{M\langle x\rangle}(x)$.

Let $H_{0}=N_{P}\left(L_{1}\right)$ be the stabilizer of $L_{1}$ in the action of $P$ on $\Delta$. Since $P$ is transitive on $\Delta, H_{0} \neq P$. Choose now a maximal subgroup $H$ of $P$ containing $H_{0}$. Since $H$ is normal in $P$ and the stabilizers of the subgroups $L_{i}$ are all conjugated in $P$, we have that $H$ contains all of them. It follows that every element $x \in P \backslash H$ has at most $k / p$ orbits on $\Delta$ and so by Lemma 3.8

$$
\lambda_{M}(x) \leq n_{p}(L)^{\frac{k}{p}} .
$$

We now consider separately the elements inside and outside $H$. As for the elements inside $H$, since $M H$ is normal in $G$, using Lemma 3.4, we get

$$
\begin{aligned}
\prod_{x \in H} \lambda_{G}(x) & =\prod_{x \in H} \frac{n_{p}(G)}{n_{p}(M H)} \lambda_{M H}(x) \\
& =\prod_{x \in H} m_{H} \lambda_{M H}(x)=\left(m_{H}\right)^{|H|} \prod_{x \in H} \lambda_{M H}(x) .
\end{aligned}
$$

Since $H$ is a Sylow $p$-subgroup of $M H$ and inequality (3) holds for $M H<G$ we get

$$
\begin{aligned}
\prod_{x \in H} \lambda_{G}(x) & =\left(m_{H}\right)^{|H|} \prod_{x \in H} \lambda_{M H}(x) \\
& \leq\left(m_{H}\right)^{|H|} n_{p}(M H)^{\frac{|H|}{p}}=m_{H}^{\frac{p-1}{p}|H|} n_{p}(G)^{\frac{|H|}{p}},
\end{aligned}
$$

where we applied (9). 
Now we turn our attention on elements in $P \backslash H$. Let $\mathcal{T}$ be a set of representatives for the right cosets of $Q$ in $P$ that are not contained in $H$. The cardinality of $\mathcal{T}$ is then

$$
|\mathcal{T}|=[P: Q]-[H: Q]=\frac{|P|-|H|}{|Q|}=(p-1) \frac{|H|}{|Q|} .
$$

We have, by Lemma 3.4

$$
\begin{aligned}
\prod_{x \in P \backslash H} \lambda_{G}(x) & =\prod_{x \in \mathcal{T}} \prod_{g \in Q} \lambda_{G}(g x) \leq \prod_{x \in \mathcal{T}}\left(\prod_{g \in Q} m_{Q\langle g x\rangle} \lambda_{M\langle g x\rangle}(g x)\right) \\
& =\prod_{x \in \mathcal{T}}\left(m_{Q\langle x\rangle}^{|Q|} \prod_{g \in Q} \lambda_{M}(g x)\right) .
\end{aligned}
$$

Now the elements $g x$ in the previous product are not in $H$ and so by (10)

$$
\begin{aligned}
\prod_{x \in P \backslash H} \lambda_{G}(x) & \leq\left(\prod_{x \in \mathcal{T}} m_{Q\langle x\rangle}\right)^{|Q|} n_{p}(L)^{\frac{k}{p}|Q||\mathcal{T}|} \\
& =\left(\prod_{x \in \mathcal{T}} m_{Q\langle x\rangle}\right)^{|Q|} n_{p}(M)^{\frac{p-1}{p}|H|} .
\end{aligned}
$$

We now want to evaluate the product $\prod_{x \in \mathcal{T}} m_{Q\langle x\rangle}$. In the following we use the bar notation for the quotients modulo $Q$. If $R=N_{M}(Q)$ we have a coprime action of $\bar{P}$ on the $p^{\prime}$-group $\bar{R}$. We apply Theorem 2.4 to this action and get

$$
\left|C_{\bar{R}}(\bar{P})\right|^{|\bar{P}| \frac{p-1}{p}}=\prod_{x \in \bar{P}} \frac{\left|C_{\bar{R}}(x)\right|}{\left|C_{\bar{R}}\left(x^{p}\right)\right|^{1 / p}} .
$$

Separating the elements inside $\bar{H}$ and those outside $\bar{H}$ and applying twice Theorem 2.4 we get

$$
\begin{aligned}
\left|C_{\bar{R}}(\bar{P})\right|^{|\bar{P}| \frac{p-1}{p}} & =\left(\prod_{x \in \bar{H}} \frac{\left|C_{\bar{R}}(x)\right|}{\left|C_{\bar{R}}\left(x^{p}\right)\right|^{1 / p}}\right)\left(\prod_{x \in \bar{P} \backslash \bar{H}} \frac{\left|C_{\bar{R}}(x)\right|}{\left|C_{\bar{R}}\left(x^{p}\right)\right|^{1 / p}}\right) \\
& =\left|C_{\bar{R}}(\bar{H})\right|^{|\bar{H}| \frac{p-1}{p}}\left(\prod_{x \in \bar{P} \backslash \bar{H}} \frac{\left|C_{\bar{R}}(x)\right|}{\left|C_{\bar{R}}\left(x^{p}\right)\right|^{1 / p}}\right) .
\end{aligned}
$$

Using the bound $\left|C_{\bar{R}}\left(x^{p}\right)\right| \leq|\bar{R}|$ and the fact that $|\bar{P} \backslash \bar{H}|=(p-1)|\bar{H}|$, we have

$$
\begin{aligned}
\prod_{x \in \bar{P} \backslash \bar{H}}\left|C_{\bar{R}}(x)\right| & =\left.\left|C_{\bar{R}}(\bar{P})^{|\bar{P}| \frac{p-1}{p}}\right| C_{\bar{R}}(\bar{H})\right|^{-|\bar{H}| \frac{p-1}{p}}\left(\prod_{x \in \bar{P} \backslash \bar{H}}\left|C_{\bar{R}}\left(x^{p}\right)\right|^{1 / p}\right) \\
& \leq\left|C_{\bar{R}}(\bar{P})\right|^{|\bar{P}| \frac{p-1}{p}}\left|C_{\bar{R}}(\bar{H})\right|^{-|\bar{H}| \frac{p-1}{p}}|\bar{R}|^{|\bar{H}| \frac{p-1}{p}} .
\end{aligned}
$$

We now observe that if $X$ is a subgroup of $P$ containing $Q$, then $Q=X \cap M$, so that $N_{M}(X) \leq N_{M}(Q)=R$ and we have

$$
\overline{N_{M}(X)}=C_{\bar{R}}(\bar{X})
$$


and since $Q \leq N_{M}(X)$

$$
m_{X}=\frac{\left|N_{M}(X)\right|}{\left|N_{M}(P)\right|}=\frac{\left|\overline{N_{M}(X)}\right|}{\left|\overline{N_{M}(P)}\right|}=\frac{\left|C_{\bar{R}}(\bar{X})\right|}{\left|C_{\bar{R}}(\bar{P})\right|}
$$

Hence, for all $x \in \mathcal{T}$,

$$
m_{Q\langle x\rangle}=\frac{\left|C_{\bar{R}}(x Q)\right|}{\left|C_{\bar{R}}(\bar{P})\right|} .
$$

Going back to our product and using (13), we then get

$$
\begin{aligned}
\prod_{x \in \mathcal{T}} m_{Q\langle x\rangle} & =\prod_{x \in \mathcal{T}} \frac{\left|C_{\bar{R}}(x Q)\right|}{\left|C_{\bar{R}}(\bar{P})\right|}=\prod_{x \in \bar{P} \backslash \bar{H}} \frac{\left|C_{\bar{R}}(x)\right|}{\left|C_{\bar{R}}(\bar{P})\right|} \\
& \leq\left|C_{\bar{R}}(\bar{P})\right|^{-|\bar{P}| \frac{p-1}{p}}\left|C_{\bar{R}}(\bar{P})\right|^{|\bar{P}| \frac{p-1}{p}}\left|C_{\bar{R}}(\bar{H})\right|^{-|\bar{H}| \frac{p-1}{p}}|\bar{R}|^{|\bar{H}| \frac{p-1}{p}} \\
& =\left|C_{\bar{R}}(\bar{H})\right|^{-|\bar{H}| \frac{p-1}{p}}|\bar{R}|^{|\bar{H}| \frac{p-1}{p}} .
\end{aligned}
$$

We now remove the bar notation using the definition of $R$ and (14),

$$
\begin{aligned}
\prod_{x \in \mathcal{T}} m_{Q\langle x\rangle} & =\left(\frac{\left|N_{M}(H)\right|}{|Q|}\right)^{-\frac{|H|}{|Q| \frac{p-1}{p}}}\left(\frac{\left|N_{M}(Q)\right|}{|Q|}\right)^{\frac{|H|}{|Q| \frac{p-1}{p}}} \\
& =\left(\frac{\left|N_{M}(Q)\right|}{\left|N_{M}(H)\right|}\right)^{\mid \frac{|H|}{|Q| \frac{p-1}{p}}} .
\end{aligned}
$$

Using this bound in inequality (12) we get

$$
\begin{aligned}
\prod_{x \in P \backslash H} \lambda_{G}(x) & \leq\left(\prod_{x \in \mathcal{T}} m_{Q\langle x\rangle}\right)^{|Q|} n_{p}(M)^{\frac{p-1}{p}|H|} \\
& \leq\left(\frac{\left|N_{M}(Q)\right|}{\left|N_{M}(H)\right|}\right)^{\frac{p-1}{p}|H|} n_{p}(M)^{\frac{p-1}{p}|H|} \\
& \leq\left(\frac{\left|N_{M}(Q)\right|}{\left|N_{M}(H)\right|} n_{p}(M)\right)^{\frac{p-1}{p}|H|}
\end{aligned}
$$

and since $Q$ is a Sylow $p$-subgroup of $M$,

$$
\prod_{x \in P \backslash H} \lambda_{G}(x) \leq\left(\frac{\left|N_{M}(Q)\right|}{\left|N_{M}(H)\right|} \frac{|M|}{\left|N_{M}(Q)\right|}\right)^{\frac{p-1}{p}|H|}=\left(\frac{|M|}{\left|N_{M}(H)\right|}\right)^{\frac{p-1}{p}|H|}
$$

By combining (11) and (15) we obtain

$$
\begin{aligned}
\prod_{x \in P} \lambda_{G}(x) & =\left(\prod_{x \in H} \lambda_{G}(x)\right)\left(\prod_{x \in P \backslash H} \lambda_{G}(x)\right) \\
& \leq\left(m_{H}^{\frac{p-1}{p}|H|} n_{p}(G)^{\frac{|H|}{p}}\right)\left(\frac{|M|}{\left|N_{M}(H)\right|}\right)^{\frac{p-1}{p}|H|} \\
& =n_{p}(G)^{\frac{|H|}{p}}\left(m_{H} \frac{|M|}{\left|N_{M}(H)\right|}\right)^{\frac{p-1}{p}|H|} .
\end{aligned}
$$


Finally, recalling (9) and the fact that $|H|=|P| / p$ we get

$$
\begin{aligned}
\prod_{x \in P} \lambda_{G}(x) & \leq n_{p}(G)^{\frac{|H|}{p}}\left(\frac{\left|N_{M}(H)\right|}{\left|N_{M}(P)\right|} \frac{|M|}{\left|N_{M}(H)\right|}\right)^{\frac{p-1}{p}|H|} \\
& =n_{p}(G)^{\frac{|H|}{p}} n_{p}(G)^{\frac{p-1}{p}|H|}=n_{p}(G)^{\frac{|P|}{p}}
\end{aligned}
$$

which is against the fact that $G$ is a counterexample.

Acknowledgements. This article is part of the author's $\mathrm{PhD}$ thesis, which was written under the great supervision of Carlo Casolo, whose contribution to this work was essential.

Thanks are also due to Francesco Fumagalli and Silvio Dolfi for his valuable comments and suggestions.

This work was partially funded by the Istituto Nazionale di Alta Matematica "Francesco Severi" (Indam).

\section{REFERENCES}

[1] C. Casolo, On the subnormalizer of a p-subgroup, J. Pure Appl. Algebra 77 (1992), no.3.

[2] C. Casolo, Subnormalizers in finite groups, Comm. Algebra 18 (1990), no. 11.

[3] G. Frobenius, Verallgemeinerung des Sylowschen Satzes, Sitzungsberichte der Preussischen Akademie, Berlin (1895).

[4] P. Gheri, Subnormalizers and the degree of nilpotence in finite groups, Proc. Amer. Math. Soc., DOI: https://doi.org/10.1090/proc/15080 (to appear in print).

[5] P. Gheri, Subnormalizers and solvability in finite groups, preprint, (2020).

[6] I.M. Isaacs and G.R. Robinson, On a theorem of Frobenius: solutions of $x^{n}=1$ in finite groups, Amer. Math. Monthly 99 (1992), no. 4.

[7] J.C. Lennox and S.E. Stonehewer, Subnormal subgroups of groups, Oxford Mathematical Monographs. Oxford Science Publications. The Clarendon Press, Oxford University Press, New York, 1987.

[8] G.A. Miller, H.F. Blichfeldt and L.E. Dickson, Theory and applications of finite groups, Dover Publications, Inc., New York 1961.

[9] G. Navarro and N. Rizo, A Brauer-Wielandt formula (with an application to character tables), Proc. Amer. Math. Soc. 144 (2016), no. 10.

[10] Speyer, David E.(1-MI) A counting proof of a theorem of Frobenius. (English summary) Amer. Math. Monthly 124 (2017), no. 4.

[11] R. Steinberg, Endomorphisms of linear algebraic groups, Memoirs of the American Mathematical Society, No. 80 American Mathematical Society, Providence, R.I. 1968.

Dipartimento di Matematica e Informatica "U. Dini", Università degli Studi di Firenze, viale Morgagni 67/A, 50134 Firenze, Italy.

E-mail address: pigheri@gmail.it 